\title{
The role of complement in the early phase of Leishmania (Leishmania) amazonensis infection in BALB/c mice
}

\author{
M.D. Laurenti ${ }^{1}$, \\ A. Örn², \\ I.L. Sinhorini ${ }^{3}$ and \\ C.E.P. Corbett ${ }^{1}$
}

\section{Correspondence \\ M.D. Laurenti \\ Departamento de Patologia \\ Faculdade de Medicina, USP \\ Av. Dr. Arnaldo, 455 \\ 1 o andar, Sala 1209 \\ 01246-903 São Paulo, SP \\ Brasil \\ Fax: +55-11-3081-7799 \\ E-mail: mdlauren@usp.br \\ Presented at the I Symposium on Advances in Medical Research, Institute of Medical Investigation Laboratories, HC-FMUSP, São Paulo, SP, Brazil, March 21-22, 2003. \\ Research supported by LIM-50, HC-FMUSP, FINEP and CAPES (PDEE 0025/95-20).}

Publication supported by FAPESP. $\ldots \ldots \ldots \ldots \ldots \ldots$

Received June 12, 2003

Accepted October 14, 2003

\author{
${ }^{1}$ Laboratório de Patologia de Doenças Infecciosas, LIM-50, \\ Departamento de Patologia, Faculdade de Medicina, Universidade de São Paulo, \\ São Paulo, SP, Brasil \\ ${ }^{2}$ Microbiology and Tumorbiology Center, Karolinska Institute, Stockholm, Sweden \\ ${ }^{3}$ Departamento de Patologia, Faculdade de Medicina Veterinária e Zootecnia, \\ Universidade de São Paulo, São Paulo, SP, Brasil
}

\begin{abstract}
Complement-depleted and -non-depleted BALB/c mice were inoculated with Leishmania (Leishmania) amazonensis promastigotes into the hind footpad to study the role of the complement system in cutaneous leishmaniasis. Total serum complement activity was measured by hemolytic assay and C3 fragment deposit at the inoculation site was determined by direct immunofluorescence in the early period of infection, i.e., at $3,24,48 \mathrm{~h}$ and 7 days post-infection. The inflammatory reaction and the parasite burden were evaluated in the skin lesion at 7 and 30 days post-infection. Total serum complement activity decreased in the early phase of infection, from 3 to $24 \mathrm{~h}$, in non-depleted mice compared to non-infected and non-depleted mice. C3 fragment deposit at the site of parasite inoculation was present throughout the period of infection in non-depleted mice. In contrast, no C3 fragment deposit was observed at the inoculation site in complement-depleted mice. Complement-depleted mice showed a significant decrease in the inflammatory response and a significant increase in the number of parasites $(70.0 \pm 5.3 v s 5.3 \pm 1.5)$ at 7 days of infection $(\mathrm{P}<0.05)$. A higher number of parasites were also present at 30 days of infection at the inoculation site of complement-depleted mice $(78.5 \pm 24.9$ vs $6.3 \pm 5.7)$. These experiments indicate that complement has an important role at the beginning of experimental cutaneous leishmaniasis caused by L. (L.) amazonensis by controlling the number of parasites in the lesion.
\end{abstract}

Key words

- Cutaneous leishmaniasis

- Leishmania (Leishmania)

amazonensis

- Complement

- Pathology

Pathology

\section{Introduction}

Leishmaniasis develops when Leishmania survives the nonspecific defense mechanism or innate immunity, such as phagocytosis by inflammatory cells and the activity of the complement system. Subsequently, the control or the progress of the lesions depends on the cell immune response.

Most reports of the role of complement in leishmaniasis have been based on in vitro experiments. The mechanisms of promastigote opsonization in normal human serum are controversial; some data indicate that 
promastigotes activate complement via the classical pathway $(1,2)$, but most reports show that Leishmania activation of complement is antibody independent by activation of lectin or alternative complement pathway $(3,4)$. Promastigotes of cutaneous strains of Leishmania are susceptible to lysis by normal serum, regardless of the presence of a specific antibody, suggesting that complement activation in vitro occurs through the alternative pathway (5). The susceptibility of the parasite to complement lysis also depends on the growth phase of the culture. Most of the promastigotes in the logarithmic phase of culture growth are lysed by the action of normal human serum, while promastigotes in the stationary phase, depending on the parasite species, may not be lysed (6). Serum resistance of infective promastigotes is not caused by their failure to bind and activate complement since both logarithmic and stationary forms have the ability to do it efficiently (7). Instead, serum resistance appears to be linked to the expression of surface antigens which differ between these two phases of parasite growth $(8,9)$. Amastigotes of cutaneous strains are more susceptible to complement lysis than visceral strains, suggesting the important role of complement factors in limiting the infection in the skin (10).

On the other hand, opsonization of parasites by fragments of complement, such as $\mathrm{iC} 3 \mathrm{~b}$ and $\mathrm{C} 3 \mathrm{~b}$, may enhance phagocytosis by specific receptors on the macrophage cell surface, which favors parasite survival, since phagocytosis through CR1 and CR3 receptors is considered to be inefficient in triggering the oxidative burst (11). In support of these data, an important role of complement in visceral leishmaniasis establishment in hamsters has been previously shown (12).

In order to study the role of the complement system in cutaneous leishmaniasis, complement-depleted and -non-depleted $\mathrm{BALB} / \mathrm{c}$ mice were inoculated subcutane- ously with promastigotes of Leishmania (Leishmania) amazonensis. Total serum complement activity and the $\mathrm{C} 3$ deposit in the skin lesion were studied together with the features of the inflammatory reaction and the parasite burden in the skin lesion during the early period of infection.

\section{Material and Methods}

\section{Animals}

Male BALB/c mice aged 8 to 10 weeks from the General Colony of the São Paulo University Medical School were kept in our laboratory during the experiments.

\section{Parasite}

Promastigotes of L. (L.) amazonensis, HSJD-1 strain, were used. Promastigotes in the stationary phase from the 3rd culture passage in supplemented RPMI 1640 medium $(10 \%$ fetal calf serum, $5 \mathrm{mM}$ HEPES, 50 $\mu \mathrm{g} / \mathrm{ml}$ gentamicin, and $100 \mathrm{U} / \mathrm{ml}$ penicillin) were harvested and washed three times in sterile saline (1500 $\mathrm{g}$ for $10 \mathrm{~min}$ ). The parasite concentration was adjusted to $2 \times 10^{8}$ promastigotes $/ \mathrm{ml}$ and $50 \mu \mathrm{l}$ was injected subcutaneously into the hind footpads of the mice.

\section{Infection}

Three groups of 25 complement-depleted $\mathrm{BALB} / \mathrm{c}$ mice and 25 non-complement-depleted BALB/c mice were inoculated with $10^{7}$ promastigotes of $L$. (L.) amazonensis by the subcutaneous route in the hind footpads. Complement depletion was induced by intraperitoneal administration of cobra venom anti-complement protein (Cobra Venom Factor, CVF) from Naja naja kaouthia (Sigma, St. Louis, MO, USA) at two doses of $6 \mu \mathrm{g} 24$ and $12 \mathrm{~h}$ before parasite inoculation. Complement depletion was maintained until $72 \mathrm{~h}$ after infection with additional doses at 0,24 
and $48 \mathrm{~h}$ after parasite inoculation. A noninfected group was used as control. Sera and fragments from the inoculation site were taken at 3, 24 and $48 \mathrm{~h}$ and 7 days after inoculation to evaluate the complement activity and $\mathrm{C} 3$ fragment deposit, respectively. Fragments of skin were taken on the 7th and 30th day of infection to evaluate the inflammatory reaction and the parasitism. Sera from non-infected BALB/c mice were collected as control for evaluation of complement hemolytic activity.

\section{Complement hemolytic titration}

The total complement activity was studied in each serum individually by the usual hemolytic assay, using triethanolamine buffer solution (TBS) (13) and the values are reported as the mean for five animals per group. Blood was collected from the retroorbital sinus by puncture, immediately immersed in ice, and centrifuged ( $200 \mathrm{~g}$ for 10 $\min$ at $5^{\circ} \mathrm{C}$ ), and the sera obtained were stored at $-80^{\circ} \mathrm{C}$ until use. The sera which presented high spontaneous hemolysis were discarded. One hundred microliters hemolysin at 1/50 dilution (anti-sheep red blood cell polyclonal antibody, produced and kindly provided by Dr. Tambourgi, Immunochemical Laboratory of Instituto Butantan, São Paulo, SP, Brazil, which reacts at up to 50 times dilution as determined by hemagglutination assay), $300 \mu 15 \times 10^{8}$ sheep red blood cell solution in TBS, $100 \mu 1$ test sera and 300 $\mu 1$ TBS, all kept in tubes in an ice bath, were added. Tubes were shaken and then incubated in a water bath at $37^{\circ} \mathrm{C}$ for $1 \mathrm{~h}$. Next, $200 \mu 1$ saline solution was added to the tubes in an ice bath and the tubes were centrifuged at $5^{\circ} \mathrm{C}(200 \mathrm{~g}$ for $10 \mathrm{~min})$. The absorbance of the supernatant solution was measured with a spectrophotometer at $541 \mathrm{~nm}$ (Micronal Protometer, São Paulo, SP, Brazil). Percent lysis was calculated by considering the positive and negative controls. One hundred microliters of distilled water was added to the positive control tube instead of $100 \mu \mathrm{l}$ test serum, and $100 \mu \mathrm{TBS}$ was added to the negative control tube instead of $100 \mu 1$ test serum.

\section{Direct immunofluorescence assay}

Complement C3 fragment deposits were evaluated by direct immunofluorescence methods using fluorescein-conjugated antimouse $\mathrm{C} 3$ goat IgG (Cappel, Aurora, OH, USA) at a concentration of $80 \mu \mathrm{g} / \mathrm{ml}$, in frozen sections of tissue obtained from the inoculation site.

\section{Histopathological studies}

The lesion and the parasitism at the inoculation site were evaluated on the 7 th and 30th day of infection by light microscopy in paraffin-embedded section stained with hematoxylin and eosin. Morphometric analysis was performed at three different levels of each section per animal using a graticule eyepiece in an area of $1 \mathrm{~mm}^{2}$ and an Olympus planapochromatic immersion objective lens (100X) (12).

\section{Limiting dilution assay for parasite quantification}

The assay was performed according to the method of Titus et al. (14) and Lima et al. (15). Skin fragments from the inoculation site between the heel and toes were taken from the footpad on the 7th and 30th day of infection, weighed and cut into several pieces in Schneider's medium (Gibco Laboratories, Grand Island, NY, USA), supplemented with $10 \%$ fetal calf serum, $2 \%$ normal human urine, $5 \mu \mathrm{g} / \mathrm{ml}$ phenol red, $5 \mathrm{mM}$ HEPES, $\mathrm{pH} 7.3,50 \mu \mathrm{g} / \mathrm{ml}$ gentamicin, and $100 \mathrm{IU} / \mathrm{ml}$ penicillin. The pieces were then homogenized vigorously using a glass tissue grinder until complete tissue disruption. The ratio for each serial dilution of the protocol was three. One hundred microliters/well from each dilution 
was dispensed into 16 wells of 96-well flatbottom culture plates. Each plate was covered with Parafilm ${ }^{\circledR}$ tightly pressed to seal each well, covered with the lid and incubated at $25^{\circ} \mathrm{C}$ for ten days. Absorbance at $450 \mathrm{~nm}$ was measured with a microtiter Multiscan apparatus (Bio-Rad, Hercules, CA, USA). Parasite growth was confirmed concomitantly under an inverted microscope (Zeiss, Jena, Germany). Data were analyzed

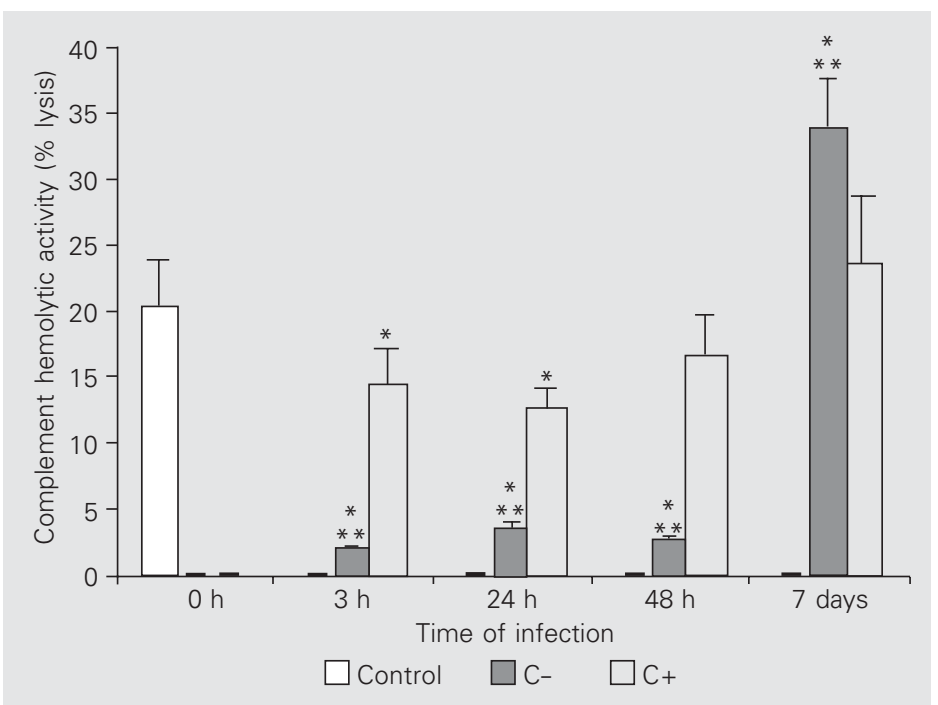

Figure 1. Complement hemolytic activity as percent lysis in the sera of complement-depleted (C-) and complement-non-depleted ( $\mathrm{C}+$ ) BALB/c mice infected with Leishmania (L.) amazonensis promastigotes. Data are representative of three separate experiments yielding similar results. Data are reported as the mean \pm SD for 5 animals per group. ${ }^{*} P<0.05$ between control and infected + non-depleted mice and between control and infected + depleted mice. ${ }^{*} P<$ 0.05 between infected + depleted and infected + non-depleted mice (one-way ANOVA).

Table 1. C3 fragment deposits at the Leishmania (L.) amazonensis subcutaneous inoculation site of complement-depleted ( $\mathrm{C}-$ ) and complement-nondepleted $(\mathrm{C}+$ ) BALB/c mice at $3,24,48 \mathrm{~h}$ and 7 days post-infection.

\begin{tabular}{lccccc}
\hline Mouse strain & Groups & \multicolumn{5}{c}{ Time post-infection } \\
\cline { 3 - 6 } & & $3 \mathrm{~h}$ & $24 \mathrm{~h}$ & $48 \mathrm{~h}$ & 7 days \\
\hline BALB/C & C- & - & - & - & - \\
& C+ & ++ & ++ & + & +
\end{tabular}

The C3 fragment deposit was analyzed by direct immunofluorescence using FITC-conjugated goat anti-mouse C3. The method showed diffuse features in the inflammatory foci and intensity was scored as negative $(-)$, mild $(+)$, moderate $(++)$ and intense $(+++)$ on the basis of the controls. Data are from three separate experiments yielding similar results. based on Poisson statistics using the ELIDA software.

\section{Statistical analysis}

The one-way ANOVA multiple range test was used with the SigmaStat Statistical Software version 1.0 to analyze the differences in complement activity in the sera and the morphometric data of the lesion between experimental groups. Differences were considered significant when $\mathrm{P}<0.05$.

\section{Results}

\section{Serum complement evaluation}

A decrease in complement hemolytic activity in the sera of mice infected with the parasite compared with non-infected controls was observed between 3 and $24 \mathrm{~h}(\mathrm{P}<$ 0.05 ; Figure 1). At $48 \mathrm{~h}$ and at 7 days of infection, the complement activity in the sera was similar to control. Complementdepleted mice compared with non-depleted mice infected with Leishmania presented very low serum complement activity at 3,24 and $48 \mathrm{~h}(\mathrm{P}<0.05)$. On the 7 th day after infection, the complement hemolytic activity was higher in depleted mice than in nondepleted and control mice.

\section{Tissue C3 fragments deposit}

The C3 fragment deposit at the parasite inoculation site was diffuse in the inflammatory foci. It was persistent and characterized by a moderate deposit between 3 to $24 \mathrm{~h}$ and mild at $48 \mathrm{~h}$ and 7 days post-infection in nondepleted mice. In complement-depleted mice, no $\mathrm{C} 3$ deposit was observed at 3, 24 and 48 $\mathrm{h}$ or at 7 days (Table 1 ).

\section{Inoculation site histopathology}

A significant decrease in the inflammatory response was observed in complement- 
depleted mice on the 7th day of infection (P $<0.05$; Figure $2 \mathrm{~A}$ ), characterized by fewer polymorphonuclear (PMN) cells and mononuclear cells compared with non-depleted mice. The number of parasites was significantly higher in depleted mice on the 7th day of infection $(\mathrm{P}<0.05$; Figure $2 \mathrm{~B})$. On the 30th day of infection, the inflammatory process was mostly characterized by mononuclear cells and was similar in both groups. However, more PMN cells were seen in complement-depleted mice. Morphometric analysis showed that parasitism was significantly increased in depleted animals $(\mathrm{P}<$ 0.05) (Figure 3A,B).

\section{Parasite quantification}

The number of viable parasites at the inoculation site was higher in complementdepleted than in non-depleted mice on the 7 th and 30th day post-infection $(\mathrm{P}<0.05$; Figure 4).

\section{Discussion}

The evolution of the infection in BALB/c mice infected with $L$. (L.) amazonensis showed a consumption of complement hemolytic activity in the sera at the beginning of the infection, between 3 and $24 \mathrm{~h}$, with

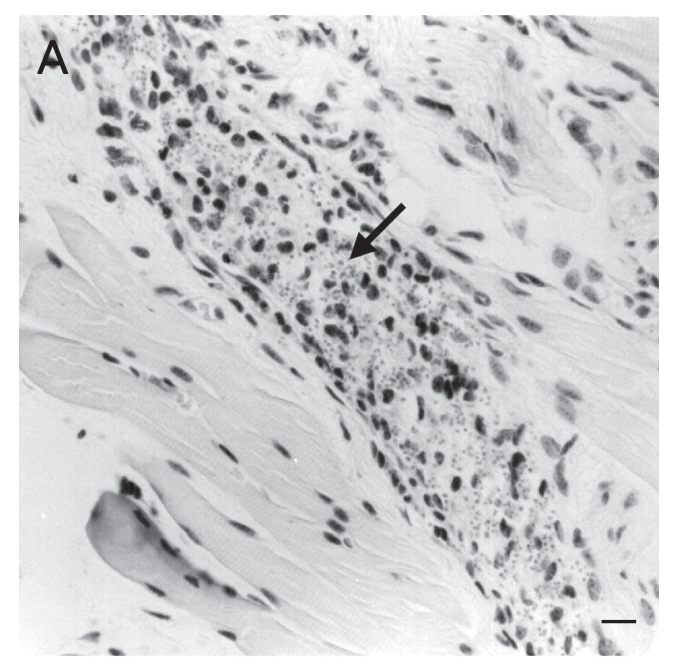

recovery from $48 \mathrm{~h}$ onwards. The presence of the $\mathrm{C} 3$ component at the subcutaneous inoculation site persisted throughout the study period, but varied from moderate to discrete. The activation of the complement system during the acute phase of Leishmania infection may contribute to resistance or to sus-
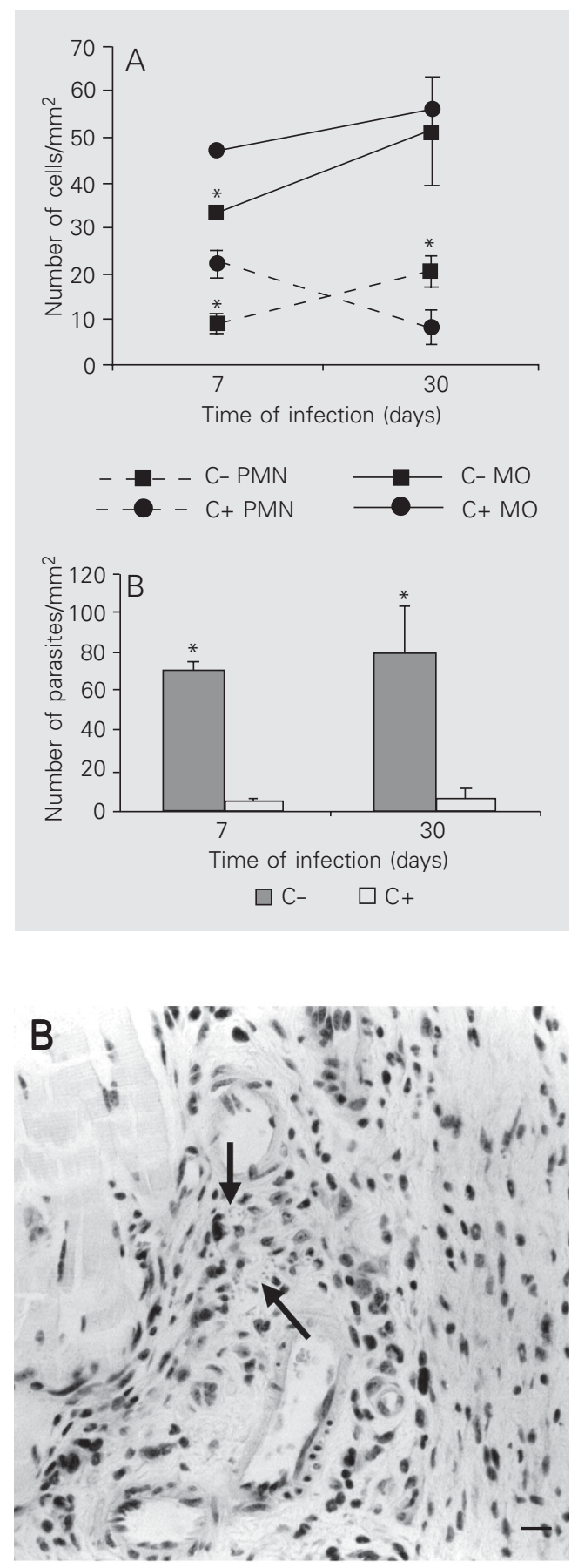

Figure 2. Morphometric analysis of the Leishmania (L.) amazonensis subcutaneous inoculation site of complement-depleted (C-) and complementnon-depleted $(\mathrm{C}+)$ BALB/C mice, showing the inflammatory reaction $(A)$ and the parasite burden of the lesion (B). Data are representative of three separate experiments yielding similar results. Data are reported as the mean \pm SD for 5 animals per group. $\mathrm{MO}=$ mononuclear cells, $\mathrm{PMN}=$ polymorphonuclear cells. ${ }^{*} P<0.05$ compared to non-depleted animals (one-way ANOVA).

Figure 3. Histopathology of an Leishmania (L.) amazonensis subcutaneous inoculation site at 7 days post-infection (H\&E, magnification 40X). A, Complement-depleted mice showing high number of parasites sorounded by inflammatory cells (arrow). B, Non-depleted mice showing few parasites in the dermal inflammatory reaction (arrows). Bars $=50 \mu \mathrm{m}$ 
ceptibility, since parasites inoculated into a vertebrate host lead to an acute inflammatory process with participation of humoral and cellular factors (12). Leishmania can activate the classical and alternative complement pathways simultaneously, in vitro. C3 binding by $L$. amazonensis promastigotes via the classical and alternative pathways is very rapid, reaching a maximum within 2 to 3 and 3 to $3.5 \mathrm{~min}$, respectively. The realtime kinetics of Leishmania promastigote lysis in normal human serum showed that lysis is an extremely rapid reaction and $10 \%$ of $L$. amazonensis promastigotes in the stationary phase showed a degree of complement resistance (16).

In vitro experiments have shown that promastigotes of the cutaneous strain of Leishmania are susceptible to lysis by normal serum by activation of the complement alternative pathway $(4,5)$. Amastigotes of cutaneous strains are more susceptible to complement lysis than visceral strains, suggesting a specific role of the complement factors limiting the infection in the skin (10). Therefore, complement activation in the early phase of $L$. (L.) amazonensis infection in $\mathrm{BALB} / \mathrm{c}$ mice in vivo, with systemic consumption and local deposit at the inoculation site, could be a factor acting in parasite destruction on the skin.

Adsorbed normal human serum shows alternative pathway $\mathrm{C} 3$ deposition capacity,

Figure 4. Number of viable parasites in the skin lesion caused by Leishmania (L.) amazonensis at 7 and 30 days after infection in complement-depleted (C-) and complement-non-depleted $(\mathrm{C}+)$ $B A L B / c$ mice. Parasites were measured by limiting dilution. Data are representative of three separate experiments yielding similar results. Data are reported as the mean \pm SD for 5 animals per group. The $95 \%$ confidence

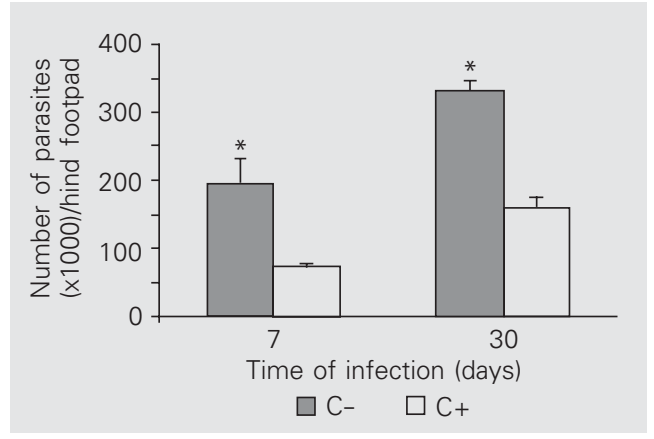

intervals showed by low limit (LL) and up limit (UL) were: 7th day of infection, (C-) LL 121.4 and UL 268.1, (C+) LL 60.5 and UL 83.5; 30th day of infection, (C-) LL 266.0 and UL 363.2, $(\mathrm{C}+)$ LL 126.9 and UL 190.9. ${ }^{*} \mathrm{P}<0.05$ compared to non-depleted BALB/c mice (one-way ANOVA).
$89.5 \%$ for $L$. amazonensis and $66.3 \%$ for $L$. donovani, similar to that seen in normal human serum-EGTA, $86.5 \%$ for L. amazonensis and $47 \%$ for $L$. donovani. Promastigotes preincubated with normal human serum-EDTA or IgM-EDTA, conditions that prevent promastigote mannan-binding lectin (MBL) and C-reactive protein binding, and subsequently incubated with adsorbed normal human serum, trigger classical pathway C3 deposition. In contrast, promastigotes preincubated with IgG-EDTA contribute only $18 \%$ of total C3 bound. These data indicate that in normal human serum, natural IgM anti-Leishmania antibodies are the main triggering factor for classical pathway activation by promastigotes, and that serum MBL and C-reactive protein do not contribute significantly to this process (16). On the other hand, MBL levels correlated directly with the probability of developing visceral leishmaniasis and affected the functions of $L$. chagasi-infected monocytes since the monocytes infected with MBL-opsonized Leishmania chagasi promastigotes secreted high levels of TNF- $\alpha$ and IL-6 (17), cytokines which can inhibit the leishmanicidal capacity of macrophages infected with $L$. mexicana $(18,19)$.

The development of the inflammatory reaction in the early phase of infection could also be considered to be a factor contributing to parasite destruction, since a large number of PMN cells migrate to the inoculation site during the first hours after infection (12). Complement is involved in the phagocytosis of Leishmania by PMN cells. In vitro experiments have shown that these cells are efficient in killing phagocytized parasites through a high oxidative burst mechanism (20).

In order to establish the role of complement in $L$. (L.) amazonensis mouse infection, mice were depleted of complement using CVF. The efficiency of CVF was confirmed by the significant decrease in complement activity in the sera when comparing depleted and non-depleted groups in the early 
phase of infection, up to $48 \mathrm{~h}$. The high complement activity by the 7 th day of infection in complement-depleted mice compared to non-depleted mice may have occurred due to a high systemic stimulus after intravascular complement activation with $\mathrm{CVF}$, which acts as $\mathrm{C} 3$-covertase leading to systemic $\mathrm{C} 3$ consumption (21). The complement activity in non-infected, complement-depleted and non-depleted animals did not show significant differences on the 7th day after CVF injection. However, the complement activity in depleted mice was slightly higher than in animals which did not receive CVF (data not shown).

C3 fragments were not detected by direct immunofluorescence in the tissue from depleted mice during the first week after infection, in spite of complement recovery in the sera by the 7 th day of infection. Morphometric analysis during the histopathologic examination of the inoculation site showed a decrease in the inflammatory response and an increase in parasite burden in depleted mice late in the acute phase, 7 days postinfection. A limiting dilution assay confirmed these results, since more viable parasites were observed in the skin of depleted animals. These data suggest that the presence of complement at the inoculation site during the early phase of infection could have a role in the control of parasite spreading in BALB/c mice infected with $L$. (L.) amazonensis, since in vitro experiments showed complement activation on the surface of $L$. amazonensis promastigotes leading to extracellular parasite lysis $(22,23)$.

For some species of Leishmania, such as $L$. major and $L$. enrietti, the complementdependent binding of promastigotes to macrophages represents the main mechanism of adhesion. In other species, such as L. amazonensis, the binding to macrophages occurs efficiently, even in the absence of serum, and the addition of exogenous complement enhances binding only slightly (24). A significant percentage of these organisms survive within the macrophages, a fact probably due to an inherent resistance of these organisms to macrophage-mediated killing mechanisms.

In order to establish the role of complement during the early phase of Leishmania infection in the evolution of disease, the histopathology and parasitism of the lesion were studied on the 30th day of infection. No differences were observed in the number of mononuclear cells between depleted and nondepleted mice, but a higher number of PMN cells was present in the lesions of complement-depleted mice. The number of viable parasites was higher in the lesions of depleted mice. The higher amount of PMN cells in the lesion of complement-depleted mice may be due to a higher parasitism, since fragments of parasites may act as a chemotactic factor for these cells (25) and may represent a late attempt by the host to destroy the parasite.

Differences between visceral and cutaneous parasite strains have been described with respect to both complement pathway activation (26) and effect of in vitro lysis by normal serum (5). In experimental visceral leishmaniasis, complement was reported to be an important factor for $L$. (L.) chagasi promastigotes to escape from the inoculation site to the viscera (12). On the other hand, the present experiments showed that complement has a role in the control of parasite spreading in the cutaneous leishmaniasis lesion caused by $L$. (L.) amazonensis infection in BALB/c mice.

\section{Acknowledgments}

We wish to thank Dr. Hiro Goto for assistance during the initial steps of the work. 


\section{References}

1. Navin TR, Krug EC \& Pearson RD (1989). Effect of immunoglobulin $\mathrm{M}$ from normal human serum on Leishmania donovani promastigote agglutination, complement-mediated killing, and phagocytosis by human monocytes. Infection and Immunity, 57: 1343-1346.

2. Dominguez M \& Toraño A (1999). Immune adherence-mediated opsonophagocytosis: the mechanism of Leishmania infection. Journal of Experimental Medicine, 189: 25-35.

3. Green PJ, Feizi T, Stoll MS, Thiel S, Prescott A \& McConville MJ (1994). Recognition of the major cell surface glycoconjugates of Leishmania parasites by the human serum mannam-binding protein. Molecular and Biochemical Parasitology, 66: 319-328.

4. Mosser DM \& Edelson PJ (1984). Activation of the alternative complement pathway by Leishmania promastigotes: parasite lysis and attachment to macrophages. Journal of Immunology, 132: 15011505.

5. Mosser DM, Burke SK, Contavas EE, Wedgwood JF \& Edelson PJ (1986). Leishmania species: Mechanisms of complement activation by five strains of promastigotes. Experimental Parasitology, 62: 394-404

6. Franke ED, McGrevy PB, Katz SP \& Sacks DL (1985). Growth cycle dependent generation of complement resistant Leishmania promastigotes. Journal of Immunology, 134: 2713-2718.

7. Puentes SM, Sacks DL, Silva RP \& Joiner KA (1990). Complement binding by two developmental stages of Leishmania major promastigotes varying in expression of a surface lipophosphoglucan. Journal of Experimental Medicine, 167: 887-902.

8. Silva RP, Hall BF, Joiner KA \& Sacks DL (1989). CR1, the C3b receptor, mediates binding of infective Leishmania major metacyclic promastigotes to human macrophages. Journal of Immunology, 143: 617-622.

9. Sacks DL, Broclin TN \& Turco SJ (1990). Developmental modification of the lipophosphoglycan from Leishmania major promastigotes during metacyclogenesis. Molecular and Biochemical Parasitology, 42: 225-234.

10. Hoover DL, Berger M, Macy CA, Hockmeyer WT \& Meltzer MS (1984). Killing of Leishmania tropica amastigotes by factors in normal human serum. Journal of Immunology, 132: 893-897.

11. Wright SD \& Siverstein SC (1983). Receptors for C3b and iC3b promote phagocytosis but not the release of toxic oxygen from human phagocytes. Journal of Experimental Medicine, 158: 20162023.

12. Laurenti MD, Corbett CEP, Sotto MN, Sinhorini IL \& Goto H (1996). The role of complement in the acute inflammatory process in the skin and in host-parasite interaction in hamsters inoculated with Leishmania (Leishmania) chagasi. International Journal of Experimental Pathology, 77: 15-24.

13. Whaley K (1985). Measurement of complement. In: Whaley K (Edi- tor), Methods in Complement for Clinical Immunologists. Butler \& Tanner Ltd., Frome and London, UK, 77-159.

14. Titus RG, Marchand M, Boon T \& Louis JA (1985). A limiting dilution assay for quantifying Leishmania major in tissue of infected mice. Parasite Immunology, 7: 545-555.

15. Lima HC, Bleyenberg JA \& Titus RG (1997). A simple method for quantifying Leishmania in tissues of infected animals. Parasitology Today, 13: 80-82

16. Dominguez M, Moreno I, López-Trascasa M \& Toraño A (2002). Complement interaction with trypanosomatid promastigotes in normal human serum. Journal of Experimental Medicine, 195: 451-459.

17. de Miranda Santos IKF, Costa CHN, Krieger H et al. (2001). Mannanbinding lectin enhances susceptibility to visceral leishmaniasis. Infection and Immunity, 69: 5212-5215.

18. Barral-Netto M, Badaro R, Barral A, Almeida RP, Santos SB, Badaro F, Pedral-Sampaio D, Carvalho EM, Falcoff E \& Falcoff R (1991). Tumor necrosis factor (cachectin) in human visceral leishmaniasis. Journal of Infectious Diseases, 163: 853-857.

19. Hatzigeorgiou DE, He S, Sobel J, Grabstein KH, Hafner A \& Ho JL (1993). IL-6 down-regulates the cytokine-enhanced antileishmanial activity in human macrophages. Journal of Immunology, 151: 36823692.

20. Pearson RD \& Steigbigel RT (1981). Phagocytosis and killing of the protozoan Leishmania donovani by human polymorphonuclear leucocytes. Journal of Immunology, 127: 1438-1443.

21. Regal JF, Frase DG, Anderson DE \& Solem LE (1993). Enhancement of antigen-induced bronchoconstriction after intravascular complement activation with cobra venom factor - Reversal by granulocyte depletion. Journal of Immunology, 150: 3496-3505.

22. Barral Neto M, Roters SB, Sherlock I \& Reed SG (1987). Destruction of Leishmania mexicana amazonensis promastigotes by normal human serum. American Journal of Tropical Medicine and Hygiene, 37: 53-56.

23. Nunes AC, Almeida Campos FR, Horta MF \& Ramalho Pinto FJ (1997). Leishmania amazonensis promastigotes evade complement killing by interfering with the late steps of the cascade. Parasitology, 115: 601-609.

24. Mosser DM \& Rosenthal LA (1993). Leishmania-macrophage interactions: multiple receptors, multiple ligands and diverse cellular responses. Seminars in Cell Biology, 4: 315-322.

25. Sorensen AL, Kharazmi A \& Nielsen H (1989). Leishmania interaction with human monocytes and neutrophils: modulation of the chemotatic response. Acta Pathologica, Microbiologica et Immunologica Scandinavica, 97: 754-760.

26. Bogdan C, Rollinghoff M \& Solbach M (1990). Evasion strategies of promastigote parasites. Parasitology Today, 14: 171-183. 